\title{
Rhizomelic Dysplasia
}

National Cancer Institute

\section{Source}

National Cancer Institute. Rhizomelic Dysplasia. NCI Thesaurus. Code C121675.

A form of skeletal dysplasia characterized by shortening of the bones of the proximal segments of the limbs (i.e. the humeri and femora). 